\title{
OPERATIVE APPROACH FOR MULTIPLE PRIMARY LUNG CARCINOMAS
}

Morihito Okada, MD

Noriaki Tsubota, MD

Masahiro Yoshimura, MD

Yoshifumi Miyamoto, MD
Of 908 patients who underwent operation for primary lung cancer between January 1985 and June 1996, we considered $57(6.3 \%)$ to have a second primary lung cancer, which was synchronous in 28 cases $(3.1 \%)$ and metachronous in 29 cases $(3.2 \%)$. Five-year survival for patients with synchronous and metachronous disease from initial treatment of cancer was $70.3 \%$ and $66.0 \%$, respectively. Survival after the development of a metachronous lesion was $32.9 \%$ at 5 years. Sixteen of the synchronous second tumors $(57 \%)$ were detected on preoperative radiography or bronchoscopy and $11(39 \%)$ at the time of operation. Survival of patients at stage I or II from treatment of a synchronous lesion $(p=0.002)$ and of a metachronous second lesion $(p=\mathbf{0 . 0 2 8})$ was significantly better compared with those at stage III or IV. Therefore it is important to carefully examine a synchronous lesion before and during the operation of a primary lung cancer and to perform close follow-up surveillance for early detection of a metachronous lesion. In treating multiple lung carcinomas consideration should always be given to performing precise staging, aggressive operative approach for early stage, and oncologically sound parenchymal sparing procedures. (J Thorac Cardiovasc Surg 1998;115:836-40)
$A^{1+n}$ lthough the idea of multiple primary lung carcinomas, which was first described by Beyreuther ${ }^{1}$ in 1924, has been generally accepted, the prevalence remains unclear and varies from $1 \%$ to $10 \%$ for synchronous and metachronous cancers together. ${ }^{2-9}$ Despite a recently reported increase, most reports of large series of patients with multiple primary lung cancers did not use developed radiographic technique, such as computed tomography, in the past. If they did it, they probably would have found a higher prevalence of synchronous cancers in the preoperative workup and of metachronous cancers during follow-up. In addition, we followed the policy that lung-saving procedures, such as bronchoplasty for a primary lesion, must always be kept in mind. This article summarizes our experience of evaluation and operative treatment of patients in whom multiple

From the Department of Thoracic Surgery, Hyogo Medical Center for Adults, Akashi City, Hyogo, Japan.

Received for publication May 21, 1997; revisions requested July 22, 1997; revisions received Nov. 17, 1997; accepted for publication Dec. 9, 1997.

Address for reprints: N. Tsubota, MD, Department of Thoracic Surgery, Hyogo Medical Center for Adults, Kitaohji-cho 13-70, Akashi City 673, Hyogo, Japan.

Copyright (C) 1998 by Mosby, Inc.

$0022-5223 / 98 \$ 5.00+0 \quad \mathbf{1 2 / 1 / 8 8 0 4 8}$ primary lung carcinomas were suspected over the past 11 years.

\section{Patients and methods}

The medical records of all patients who underwent operation for primary lung cancer at our center between January 1985 and June 1996 were reviewed. All charts of patients with a probable diagnosis of multiple primary lung cancer were available for analysis. The following criteria, based on and modified from those of Martini and Melamed, ${ }^{4}$ have been used for designation of multiple primary lung cancers: (1) The tumors were anatomically separate (different lobes or lungs). (2) The tumors were histologically different. If the tumors were histologically the same, they must have no systemic metastases or mediastinal spread. Where all the lesions were squamous cell carcinomas, a classification was accepted if a separate origin from an area of carcinoma in situ could be identified for each. In case of the metachronous tumors with the same histologic findings, tumor-free interval was at least 2 years. Patients who did not meet these criteria were excluded. All cancers were staged using the criteria of the New International Staging System for Lung Cancer, ${ }^{10}$ and staging was based on data obtained from imaging, bronchoscopy, invasive diagnostic techniques, operative findings, and pathologic findings. Careful intraoperative staging at the time of operative resection was done by dissecting intrapulmonary, hilar, and mediastinal nodes. Histologic typing was done according to the World Health Organization classification. ${ }^{11}$ All cases of bronchioloalveolar carcinoma were excluded just because of the question of multicentricity of those primary cancers. A syn- 
Table I. Histologic classification of multiple primary lung carcinomas

\begin{tabular}{lcc}
\hline \multicolumn{1}{c}{ Classification } & Synchronous & Metachronous \\
\hline Squamous cell carcinoma & & \\
Squamous cell carcinoma & $6(21)$ & $14(48)$ \\
Adenocarcinoma & $12(43)$ & $2(6)$ \\
Small cell carcinoma & 0 & $2(6)$ \\
Carcinoid & $1(4)$ & 0 \\
Adenosquamous carcinoma & $1(4)$ & 0 \\
Large cell carcinoma & 0 & $1(3)$ \\
Adenocarcinoma & & \\
Adenocarcinoma & $6(21)$ & $5(17)$ \\
Small cell carcinoma & $1(4)$ & $3(10)$ \\
Large cell carcinoma & $1(4)$ & $1(3)$ \\
Small cell carcinoma & & \\
Large cell carcinoma & 0 & $1(3)$ \\
Total & $28(100)$ & $29(100)$
\end{tabular}

Numbers in parentheses are percentages.

chronous multiple primary lung cancer was identified if there was radiographic evidence of a second primary tumor before the first operation or if a second primary tumor was discovered incidentally during operation or in the resected specimen. The larger of two synchronous tumors was considered the first primary cancer for the purpose of analysis. All patients who underwent resection for a primary lung cancer had a chest roentgenogram, a history, and a physical examination at the follow-up visit, which took place at least twice a year.

Cumulative survivals were calculated by the KaplanMeier estimation, and differences in survival were determined by log-rank analysis.

\section{Results}

From January 1985 to June 1996, 908 patients underwent operation for a primary lung cancer at our center. Fifty-seven (6.3\%) patients were found to have a second primary lung cancer, three of these also had a third cancer. ${ }^{12}$ Synchronous tumors comprised 28 of $908(3.1 \%)$ and metachronous tumors 29 of 908 (3.2\%). Overall follow-up ranged from 6 to 144 months, with a median of 51 months.

Synchronous carcinoma. The average age at initial treatment was $64.3 \pm 7.7$ years (mean \pm standard error), with an age range of 47 to 76 years. There were 24 male and 4 female patients. Squamous cell carcinoma constituted 14 of $28(50 \%)$ of the first tumors and 12 of $28(43 \%)$ of the second tumors. Adenocarcinoma was seen in 11 of 28 (39\%) of the first tumors and 15 of $28(54 \%)$ of the second tumors. The histologic findings of the synchronous second tumor were the same as those of the synchronous first tumor in $12(43 \%)$ patients and equally consisted of squamous cell carcinoma and adenocarcinoma (Table I). Sixteen of the sec-
Table II. Staging of multiple primary lung carcinomas

\begin{tabular}{ccccc}
\hline & \multicolumn{2}{c}{ Synchronous } & & \multicolumn{2}{c}{ Metachronous } \\
\cline { 2 - 2 } \cline { 5 - 5 } Stage & Most advanced tumor & & First tumor & Second tumor \\
\hline I & $11(39)$ & & $15(52)$ & $17(59)$ \\
II & $5(18)$ & & $6(21)$ & $1(3)$ \\
IIIA & $5(18)$ & & $8(28)$ & $11(38)$ \\
IIIB & $3(11)$ & & 0 & 0 \\
IV & $4(14)$ & & 0 & 0 \\
Total & $28(100)$ & & $29(100)$ & $29(100)$ \\
\hline
\end{tabular}

Numbers in parentheses are percentages.

ond tumors $(57 \%)$ were detected on preoperative radiography or bronchoscopy, and $11(39 \%)$ were discovered during operation. In the other remaining patient (4\%), the second lesion was diagnosed incidentally by histologic examination of the resected specimen. The second tumor was on the same side as the first tumor in 21 cases (75\%). Stage I, II, IIIA, or IIIB disease was noted in $11(39 \%), 5(18 \%), 5$ $(18 \%)$, or 3 tumors $(11 \%)$, respectively (Table II). The four remaining patients $(14 \%)$ had double tumors that were histologically different, and postoperative pathologic examination showed additional lesions that were diagnosed with pulmonary metastasis. Staged resection was performed on $18 \%$ of synchronous lesions (5 of 28) because they were contralateral to the first tumor. Resection was performed in all patients except for one with extended disease. Lobectomy was by far the most common procedure and was performed in 23 patients $(82 \%)$, including seven combined sleeve resections of the bronchus. Two pneumonectomies including sleeve technique for the bronchus and two segmentectomies were carried out. Resection of the tumors was possible in all patients, but two patients had pleural dissemination. No postoperative deaths occurred within 30 days after resection.

Overall 5-year survival was $70.3 \%$ for all patients having a synchronous cancer (Fig. 1). The 5-year survival of patients with stage I or II and stage III or IV was $79.0 \%$ and $41.0 \%$, respectively. Survival for patients with stage I or II was significantly better $(p=0.002)$ compared with stage III or IV (Fig. 1).

Metachronous carcinoma. The average age at the time of treatment for the first and second tumor was $62.6 \pm 8.6$ years (mean \pm standard error) with an age range of 32 to 73 years, and $66.7 \pm 9.3$ years with an age range of 33 to 79 years, respectively. All patients except one were men. The mean interval between the initial treatment and the second was 


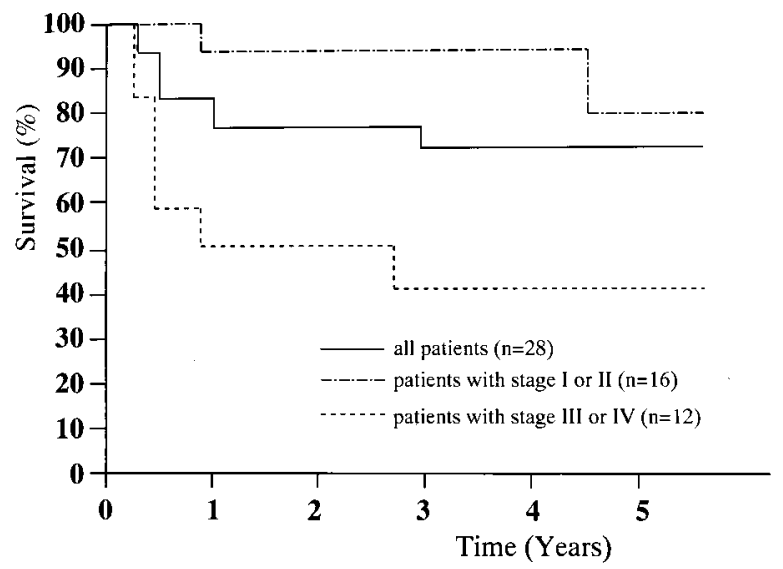

Fig. 1. Actuarial survival curves of patients in whom synchronous multiple primary lung cancers developed. Middle curve indicates survival for all patients with synchronous disease. Top and bottom curves indicate the survival of patients with stage I or II and stage III or IV, respectively. Survival of patients with stage I or II was significantly better $(p=0.002)$ compared with stage III or IV.

$4.1 \pm 2.3$ years. The longest interval was 10 years and 8 months, and the shortest only 8 months. Every case whose interval was less than 2 years had a histologically different tumor. More than 5 years after the first tumor, metachronous primary tumors appeared in 13 patients $(45 \%)$. Squamous cell carcinoma was the most common histologic type, making up $17(59 \%)$ of 29 patients as the first tumor and $16(55 \%)$ patients as the second tumor. The next most common histologic type was adenocarcinoma, occurring in $8(28 \%)$ and $8(28 \%)$ of the first and second tumors, respectively. The first and second tumors shared the same histologic findings in 19 $(63 \%)$ patients (Table I). The same histologic type was more likely to occur if the first tumor was a squamous cell carcinoma as opposed to an adenocarcinoma (14 of 17 patients, $82 \%$ and 5 of $8,63 \%$, respectively). Stage I in first and second tumors constituted 52\% (15 of 29) and 59\% (17 of 29), respectively. No tumors were more advanced than stage IIIA (Table II). Resection of the tumors was possible in all patients. Obviously, more patients underwent segmentectomy or wedge resection at reoperation for the second disease compared with initial operation. No postoperative deaths occurred within 30 days after resection.

The 5-year actuarial survival after the initial and the second resection was $66.0 \%$ and $32.9 \%$, respectively, for all patients with a metachronous cancer

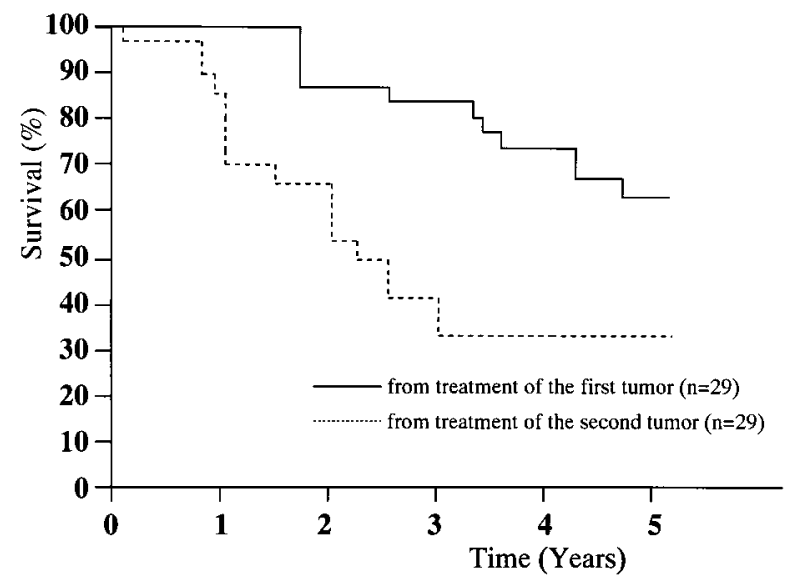

Fig. 2. Actuarial survival curves of patients in whom metachronous multiple primary lung cancers developed. Top and bottom curves indicate the survival from treatment of the first and the second tumor, respectively.

(Fig. 2). Survival for patients with metachronous disease determined from treatment of first tumors was not significantly different from that of patients with synchronous disease. Survival curves from treatment of the first tumor indicated no significant difference between patients with stage I or II and stage III (Fig. 3). Patients with stage I or II and stage III of the second tumor had survival rates of $49.8 \%$ and $24.0 \%$ for 5 years, respectively. Survival from treatment of the second tumor was significantly better $(p=0.028)$ for patients with stage I or II compared with stage III (Fig. 4).

\section{Discussion}

Clinically, it is difficult to distinguish a second primary carcinoma from a metastatic lesion arising from a first tumor. Martini and Melamed ${ }^{4}$ in 1975 outlined the criteria for differentiation between the two, which have been used by most subsequent authors, including us. Pairolero and associates ${ }^{13}$ using criteria similar to ours, reported a $52 \%$ 2-year survival for metachronous primary tumors compared with 23\% and 9\% 2-year survival for local and distant recurrent disease, respectively. These data suggest that patients with multiple primary tumors have a more favorable prognosis than patients with locally recurrent or metastatic disease. ${ }^{14,15}$ Therefore an appropriate identification of multiple primary lung cancers is critical. In addition to the application of stringent criteria for the diagnosis of multiple primary lung cancers, incidence of this disease may be affected by several factors, including 


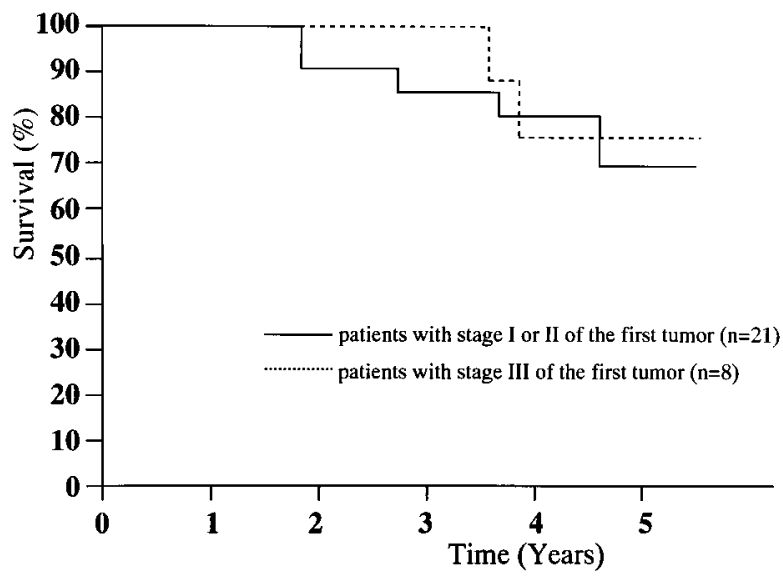

Fig. 3. Actuarial survival curves of patients in whom metachronous multiple primary lung cancers developed from treatment of the first tumor. Solid and dashed lines indicate the survival in patients with stage I or II and stage III of the first tumor, respectively. No significant differences in survival probability were found between stage I or II and stage III.

the sensitivity of diagnostic procedures, the pathologist's skill, the efficacy of the treatment used, and the length of the patient's survival. These latter factors, in particular the development of radiography, would contribute to an increase in the incidence of these multiple tumors, and therefore standardized criteria for the diagnosis of this disease should be developed. In contrast to recent years, most studies in the past did not use computed tomography. If they did it preoperatively, they could have found a higher prevalence of synchronous tumors in the same way they might have found a higher prevalence of metachronous cancers or might have detected them earlier should they have used it during follow-up. The developed radiographic technique in this series probably accounted for the increased incidence of multiple primary tumors and a high proportion of synchronous lesions.

The result that $57 \%$ of synchronous second lesions were discovered preoperatively and $39 \%$ intraoperatively indicated the role of careful detection of second lesions before and during the procedure for a primary lung cancer. The inferior survival of metachronous second lesions compared with first lesions might be related to underestimating because of technical difficulties during the second procedure and to resorting to lesser resections because of limited pulmonary reserve. The survival advantage of metachronous lesions compared with synchro-

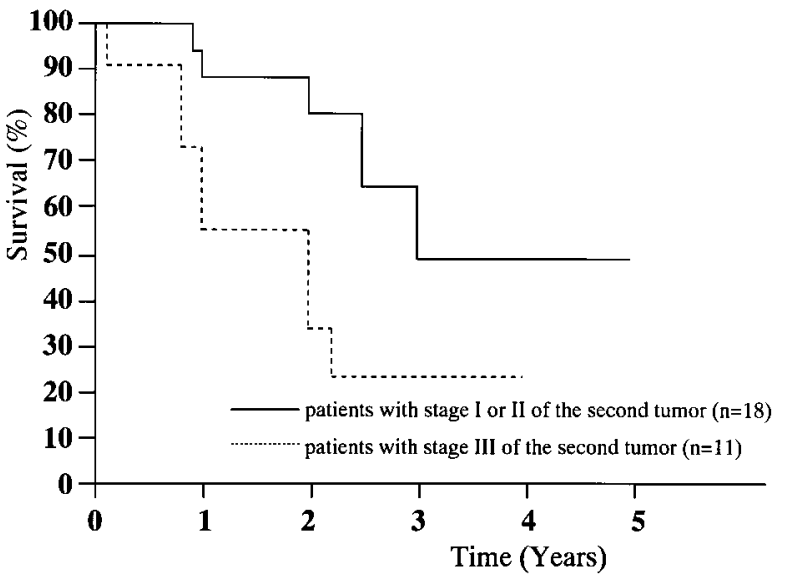

Fig. 4. Actuarial survival curves of patients in whom metachronous multiple primary lung cancers developed from treatment of the second tumor. Solid and dashed lines indicate the survival in patients with stage I or II and stage III of the second tumor, respectively. Survival of patients with stage I or II was significantly better $(p=$ 0.028) than that of patients with stage III disease.

nous ones has been reported by others ${ }^{14,16,17}$ and does not agree with our study. The possibility that some patients with recurrent or metastatic disease have also been included as subjects cannot be ruled out for other published literature. We believe that the difference between synchronous and metachronous cancers is just an arbitrary one because it refers to the moment of diagnosis and not to the moment of development.

The question of whether a newly discovered lung lesion after resection is actually a second primary tumor or a recurrence remains a theoretical one. We follow the policy of thoroughly restaging all such lesions and operating with the intention to cure whenever possible. Therefore, although patients with unilateral lesions found in separate lobes should be treated by pneumonectomy or bilobectomy, lobectomy with additional wedge resection may be done in patients with limited pulmonary function. In patients with bilateral lesions a curative resection should be done on the side that initially appears to be more advanced. However, lung tissue should not be sacrificed for expediency in these patients. If a lung-saving procedure such as a sleeve lobectomy for the first lesion is done and sufficient pulmonary function is preserved, a second radical operation is possible. Otherwise, there is no choice except to perform conservative treatment, including wedge resection, chemotherapy, radiation therapy 
or all three. A curative yet limited extent of operation allowing for maximum preservation of pulmonary function should be of prime consideration. This strategy may allow subsequent treatment of the second lesion by operation. Bronchoplasty, including sleeve resection, has been used frequently at this center not only for patients with limited pulmonary reserve but also for those with sufficient reserve. ${ }^{18}$ Bronchoplasty on the first occasion would leave the patient with more lung tissue to resect should a second primary tumor develop later. This principle of conservation is very important because the loss of pulmonary function from more radical procedures for the first lesion may be extensive or may prevent a second operation. Re-resection can almost be performed safely, as indicated by our series with no operative deaths and others' operative mortality rates. ${ }^{2,16}$

Survival for patients with early-stage multiple primary tumors was significantly better than those with advanced tumors, as has been reported by us and others. ${ }^{2}$ These data highlight the need for careful lifelong screening for a second lesion to improve overall survival. We obtained very poor results in the whole group of stage III or IV synchronous tumors and of stage III metachronous second tumors. The advance of staging and the presence of a second primary tumor could influence survival. Therefore, when a new primary lung tumor has been found, precise staging is necessary and an aggressive operative approach is indicated, especially for earlier second tumors.

We recommend that the resection be tissuesparing whenever it is reasonable to do so whether it is the first or any subsequent operation. Therefore we should avoid pneumonectomy and instead try to use bronchoplasty as much as possible.

\section{REFERENCES}

1. Beyreuther H. Multiplicitat von Carcinomen bei einem Fall von sog: "Schneeberger" Lungenkrebs mit tuberkulose. Virchows Arch 1924;250:230-43.
2. Van Bodegam PC, Wagenaar SS, Corrin B, Baak JPA, Berkel J, Vanderschueren R. Second primary lung cancer: importance of long term follow up. Thorax 1989;44:788-93.

3. Shields TW, Humphrey EW, Higgins GA, Keehn RJ. Longterm survivors after resection of lung carcinoma. J Thorac Cardiovasc Surg 1978;76:439-45.

4. Martini N, Melamed MR. Multiple primary lung cancers. J Thorac Cardiovasc Surg 1975;70:606-12.

5. Rohwedder JJ, Weatherbee L. Multiple primary bronchogenic carcinoma with a review of the literature. Am Rev Respir Dis 1974;109:435-45.

6. Razzuck MA, Pockey M, Urschel HC, Paulson DL. Dual primary bronchogenic carcinoma. Ann Thorac Surg 1974;17: 425-33.

7. Smith RA, Nigam BK, Thompson JM. Second primary lung carcinoma. Thorax 1976;31:507-9.

8. Boucot KR, Weiss W, Cooper DA. Second pulmonary neoplasms among long-term survivors of lung cancer. Am Rev Respir Dis 1965;92:767-70.

9. Shields TW. Multiple primary bronchial carcinomas. Ann Thorac Surg 1974;27:1-2.

10. Mountain CF. A new international staging system for lung cancer. Chest 1986;89:225S-33S.

11. World Heath Organization histologic typing of lung tumours. 2nd ed. Am J Clin Pathol 1982;77:123-36.

12. Okada M, Tsubota N, Yoshimura M, Kubota M, Murotani A. Simultaneous occurrence of three primary lung cancers. Chest 1994;105:631-2.

13. Pairolero PC, Williams DE, Bergstalh EJ, Piehler JM, Bernatz PE. Postsurgical stage I bronchogenic carcinoma: morbid implications of recurrent disease. Ann Thorac Surg 1984;38:331-6.

14. Deschamps C, Pairolero PC, Trastek VF, Payne WS. Multiple primary lung cancers: results of surgical treatment. J Thorac Cardiovasc Surg 1990;99:769-78.

15. Watanabe Y, Shimizu J, Oda M, Tatsuawa Y, Hayashi Y, Iwa T. Second surgical intervention for recurrent and second primary bronchogenic carcinomas. Scand J Thorac Cardiovasc Surg 1992;26:73-8.

16. Mathisen DJ, Jensik RJ, Faber P, Kittle CF. Survival following resection for second and third primary lung cancers. J Thorac Cardiovasc Surg 1984;88:502-10.

17. Ferguson MK, DeMeester TR, Deslauriers J, Little AG, Piraux M, Golomb H. Diagnosis and management of synchronous lung cancers. J Thorac Cardiovasc Surg 1985;89: 378-85.

18. Tsubota N, Yoshimura M, Murotani A, Miyamoto Y, Matoba Y. Atypical bronchoplasty to lung cancer and benign bronchial disease. Tohoku J Exp Med 1994;172:209-20. 